\title{
An Overview of Fall-Related Systems Developed in Canes*
}

\author{
Pedro Mouta $^{1}$, Nuno Ferrete Ribeiro ${ }^{1}$, Luís Gonçalves ${ }^{1}$ and Cristina P. Santos ${ }^{1}$
}

\begin{abstract}
The number of falls is growing as the main cause of injuries and deaths in the geriatric community. As a result, also the cost of treating the injuries associated with falls is increasing. Thus, the development of fall detection and prediction systems with the capability of real-time monitoring without user restriction is imperative. For this reason, canes can be a great choice to embed fall-related systems. This paper aims to achieve a complete state-of-the-art concerning the development of fall detection and prediction algorithms implemented in canes. In order to accomplish this goal, a comprehensive search was performed. This search includes the IEEE, Scopus and Web of Science databases regarding developed systems in the literature. Also, a commercial search on Google and a patent search on Espacenet and the United States Patent and Trademark Office was performed. To this day, it has not been developed any type of cane related system which focuses on the temporal window preceding a fall and that accesses the risk of falling, even though a considerable amount of fall detection systems have already been presented.
\end{abstract}

\section{INTRODUCTION}

World Health Organization stated that falls are the second main reason of death by accident worldwide, representing not only one of the main unwanted accidents, but also a challenge to patient safety and, therefore, their care quality [1]. According to the International Database of the U.S. Census Bureau, in 2015, the typical proportion of individuals older than 65 years in developed countries was roughly $17 \%$. This proportion is expected to reach $30 \%$ in 2050 which is very alarming since one-third of the elderly suffer at least one fall each year, resulting frequently in serious health complications. Thus, falls in older adults are a common and increasing health problem [2], [3].

For instance, in the United States of America, the estimated medical costs concerning falls in 2015 were, approximately, $\$ 32$ billion [2]. By 2020, expenses linked to injuries from falls to senior citizens are expected to cost roughly $\$ 43.8$ billion [4]. Moreover, according to Lachtar et al. [5], the annual average cost is $\$ 20.000$ per individual due to injuries from a fall in the United States also.

Thus, in order to fight these costs, the scientific community has strongly focused on Fall Prediction (FP), Fall Risk

\footnotetext{
*This work has been supported by the FCT - Fundação para a Ciência e Tecnologia - with the scholarship reference PD/BD/141515/2018, by the FEDER funds through the COMPETE 2020 - Programa Operacional Competitividade e Internacionalização (POCI) and P2020 with the Reference Project EML under Grant POCI-01-0247-FEDER-033067, and through the COMPETE 2020 POCI with the Reference Project under Grant POCI-010145-FEDER-006941.

${ }^{1}$ P. Mouta, N. F. Ribeiro, L. Gonçalves and C. P. Santos are with Center for MicroEletroMechanical Systems (CMEMS), University of Minho, 4800-058, Guimarães, Portugal pmfmouta@gmail.com, nuno.ferrete.ribeiro@gmail.com,

lgoncalvesddei.uminho.pt and

cristina@dei.uminho.pt
}

Assessment (FRA) and Fall Detection (FD). FP systems combine data from multiple sensors with the objective of alerting the user prior to a fall in a various number of circumstances and scenarios. As a result, they are able to prevent the bad consequences of a fall [6].

FRA identifies people of high fall risk upon specific and protocoled interventions. Three types of assessment are relevant which enable the association of the subjects fall risk with specific interventions. The assessment can be accomplished by health practitioners involving the use of scales or tools (e.g. STRATIFY), of functional mobility assessments and the use of the patients fall history, strength, balance and gait [7]. FD systems focus on developing methods in which a fall is automatically detected, and an alarm is triggered. Nevertheless, the wearable system will weight on the individual and hinder its flexibility [8].

Consequently, the use of accessories where elderly may take with or wear on, e.g. necklaces, watches or canes, can be a serious alternative. However, using these accessories to continuously monitor the subject involves a considerably sized battery. Therefore, the use of smaller devices will result in a low power supply for a short amount of time, which is not optimal. The constant hand movement is also a factor to discard its use which is too high to monitor for long periods of time. Since the installation of sensors on everyday objects for fall detection and prediction is desirable, canes are a good choice due to their size, which is usually big enough to embed a large battery, and because they are commonly used by the geriatric community [9]. Nowadays, more than 4 million people in the United States alone use a cane and its usage is expected to increase since the elderly community is growing and device ownership increases with age [10], [11].

By reducing weight supported on the individual legs, canes can ease the pain related from injuries or clinical pathologies such as hip fracture, or compensate for weakness or impaired motor control of the leg [10]. Canes increase the person stability by widening the base of support, reducing the weight load on the lower limbs, and giving the user a sense of safety which results in a lower fall risk [11].

The aim of this paper is to achieve a complete state-of-theart concerning the development of fall detection and prediction related systems implemented in canes. The remainder of this paper is organized as follows. Section II summaries the related work found in the literature regarding cane systems with embedded fall detection and prevention methodologies, as well the search strategy and eligibility criteria applied. Section III gives an overview of commercial canes available on the market. Section IV contains a patent review conducted similarly as the one used in the Preferred Reporting Items 
for Systematic Reviews and Meta-Analyses (PRISMA) flow diagram. Finally, in section V, the paper is concluded based on the researched performed and results found.

\section{CANES IN LITERATURE}

\section{A. Search Strategy \& Eligibility Criteria}

A comprehensive search was accomplished in order to understand the following topics: i) what fall-related strategies are implemented with canes; ii) how canes are instrumentalized; iii) how and what algorithms are implemented; and iv) what researchers did to validate their system in order to develop a cane with embedded fall detection and/or prediction.

On October 9th, 2018, the search was completed in the IEEE, Scopus and Web of Science databases with the keywords (Cane OR "Walking Stick") AND ("Near Fall" OR "Fall Detection" OR "Fall Prediction" OR "Fall Prevention" OR "Falling"). A total of 325 articles were found. To decide which ones were most relevant, articles were selected based on whether if the system has implemented fall detection/prediction mechanisms with built-in technology into the cane. Nine articles remained for this state-of-the-art based on the abovementioned eligibility criteria.

\section{B. Search Results}

All the studies found implemented fall detection systems and only two authors also included fall prevention strategies. Di et al. [12]-[15], and Yan et al. [16], tried to avoid falls by using a cane robot with an omni-wheel base different from the other instrumented canes.

In 2011, Di et al. designed a system that with the subjects Center of Gravity (COG), an impedance control system moved the cane to equal the subjects fall direction. However, the experimental protocol did not include falls and its results were not disclosed [15].

Later, in 2013, another prototype based on the Zero Moment Point (ZMP) was developed. When the ZMP surpasses the support polygon, a fall is eminent. With the same fall prevention mechanism as before, [15], the ZMP is able to remain inside the boundaries. In this work, the experimental protocol and results are not revealed [13], [14].

For last, in 2016, the same research team realized a comparison between the employment of two different algorithms for fall detection based on a real-time calculation of the individual's Center Of Pressure (COP) and its leg motion which obtained an accuracy of $75 \%$ and $91,2 \%$ respectively. Regarding the fall prevention mechanism, an impedance control system similar to the ones previously described was implemented. [12].

Yan et al. [16], developed a cane-type walking-aid robot in which their system was based in the Human-Robot Coordination Stability. Although the results were not uncovered, they concluded that the system provided a new way for fall detection and fall prevention.

Excluding robotic systems with an omni-wheel base, [12][16], and wearable foot sensors [12]-[15], generally, contact and triaxial inertial sensors are the most common sensors embedded into canes [5], [17], [18]. More specifically, Accelerometers (Acc), Gyroscopes (Gyr), Magnetometers (Mag) and Force Sensitive Resistors (FSRs) with a sampling frequency between 15 and $100 \mathrm{~Hz}$. Their location in the cane can be in one of four places, near the canes handle [17], [18], into the handle [12]-[14], [16], [17], in its base [5], [13], [14], [16], [17], [19], or in the cane's body at knees level [12]-[14]. As far as the location of the wearable sensors, they are allocated in three places, in the waist [13], [14], ankle [13], [14] and the sole of the feet [12]-[14]. As result, information about sensors used on canes and their location were combined in Fig.1.

Concerning the implemented algorithms, it is possible to say that the strategies can be considered as complete in terms of low-power consumption, considering that almost all developed systems implemented threshold-based algorithms of the acceleration data for classification [5], [18], [19]. After the collection of enough acceleration data from different fall directions, thresholds are computed, and when its values exceed a single or several thresholds in a specific sequence over a time period, a fall is detected. Yet, this method is prone to give false positive outcomes to many exceptional scenarios. As a result, the tendency of algorithms used in fall-related systems is to increase the number of devices with embedded machine learning algorithms [8]. Nevertheless, its implementation in canes is yet to be completed.

From the acceleration data acquired, features can be extracted from the signal in the time domain. The Sum Vector Magnitude (SVM) is the most commonly computed feature [17]-[19], because the fall direction and the posture of the subject are almost impossible to predict considering there is not a pattern observed in a single acceleration axis. When the acceleration data from all the different axis is summed, it is observed a pattern describing the different stages of a fall [18].

In regard to the experimental protocol and subject's information, only half of the systems disclosed it. All systems were tested with healthy subjects [12], [17], [18], excluding [5], which is not ideal since the target population is the elderly community.

The fall direction accounted in the systems validation protocol vary. Lan et al. [17], and Lachtar et al. [5], divide falls into forward, backward and sideward direction. However, Chen et al. [18], only consider forward and backward falls. Yan et al. [16], and Di et al. [12], only account for forward falls. The detection rate of the systems can achieve between $84 \%$ (forward and backward falls), [18], and 100\% (forward falls) [5], [17].

\section{COMMERCIAL CANES}

Even though fall prediction systems are relatively new regarding canes, fall detection systems have been employed for a considerable amount of time as we can see from the bibliographical search above. After an extensive search for commercial canes with fall-related embedded systems, it was possible to find two products namely: iStand $\mathrm{SmartCan}^{T M}$ and Dring Smartcane, yet, only the first is available for sale. 


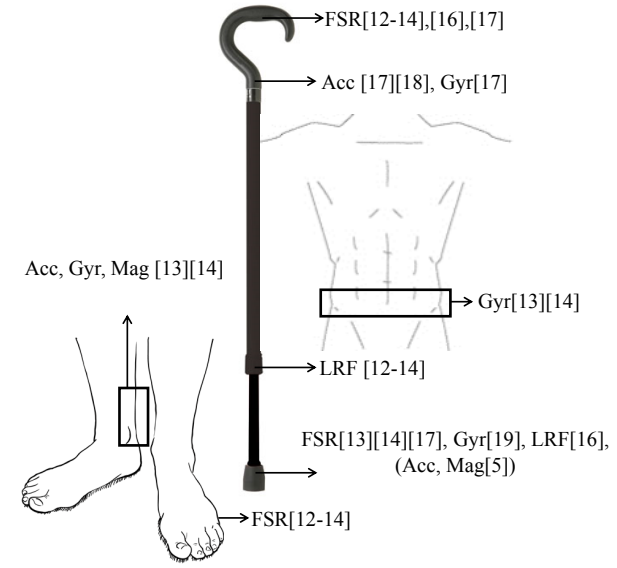

Fig. 1: Four canes locations and three locations on the human body where sensors are attached.

\section{A. iStand SmartCan ${ }^{T M}$}

The iStand Smart Cane is a device manufactured by WhatBox, Inc., that offers families a trackable cane with GPS and a fall detection system. The device has Bluetooth capabilities which allow it to pair with the iStand cane mobile application, ending the communication gap and allowing real-time visibility for family, friends and caregivers. All notifications are sent through Facebook or text messages in the event of a lost cane, low battery, or fall detection. Every cane has a flexible shaft for joint comfort and an anti-trip base that stands alone. The device is currently available only in the United States for \$99 [20].

\section{B. Dring Smartcane}

The Dring Smartcane from French start-up Nov'in has a built-in GPS, Acc and Gyr in order to track the individual movements. In case of a fall, the cane sends an alert to a selected caregiver. In order to enable the adaption of every device to a specific user, artificial intelligent algorithms were implemented to process the data which helps understand a user's habits and movements. The release date of the device is yet to be disclosed [21].

\section{PATENT REVIEW}

\section{A. Search Strategy}

On October 10th, 2018, an advanced patent search on international patents was performed on Espacenet (http://spacenet.com), which allows free access to over 100 million international inventions and technical developments. The search was conducted based on the title and abstract, the keywords used were (Cane OR "Walking Stick") AND ("Fall" OR "Near Fall" OR "Fall Detection" OR "Fall Prediction" OR "Fall Prevention" OR "Falling"). The selection of patents was based on available schemes, and appropriate titles and abstracts. On October 11th, 2018, the previous procedure was also performed this time on the United States Patent and Trademark Office (http://patft.uspto.gov). The keywords utilized were the same as the abovementioned search.

\section{B. Search Results}

Regarding the search process accomplished, a total of 17 patents were selected in the end. On Espacenet, 404 patents were found, and only 50 were selected based on their title. In turn, 6756 patents were found on the United States Patent and Trademark Office, where 25 patents were selected similarly as Espacenet. Ultimately, from the 75 patents selected, 17 were included based on abstract, description and drawings. Fig. 2 illustrates a flow diagram of the entire study selection process.

From all the 17 patents, 3 were selected based on the following criterion: whether it has fall detection/prediction mechanisms and whether it has only built-in technology into the cane. The results can be seen in Table I.

From the selected patents, all present prediction mechanisms. The patent regarding the anti-falling walking stick comprises a walking stick shell, a supporting unit, a threedimensional axial accelerated speed monitor and a central analyzing unit. When the subject walks unstably, is about to fall or even its already falling, a supportive leg can pop up automatically. Hence, the user can be successfully supported and can avoid injuries. Since a person can sustain falls in different directions, the central analysis unit analyses the received data and determines the direction of the fall. Thus, the two branches closest to the fall direction pop up [22].

The robotic cane device includes a grip handle equipped with FSRs, a cane body extending from the grip handle, a motorized omni-directional wheel, a balance control sensor and a controller module. With the data acquired and computed, the omni-directional wheel will attempt to retain the robotic cane in a substantially upright position. As regards to the fall prediction mechanism, if the grip force value surpasses the set threshold, the system quickly provides a counter force from the user's weight projection [23].

Finally, patent number 3 comprises an omni-wheeled base with a fall prevention mechanism linked to the computation of the subjects ZMP much like Di et al. [13], [14], [24].

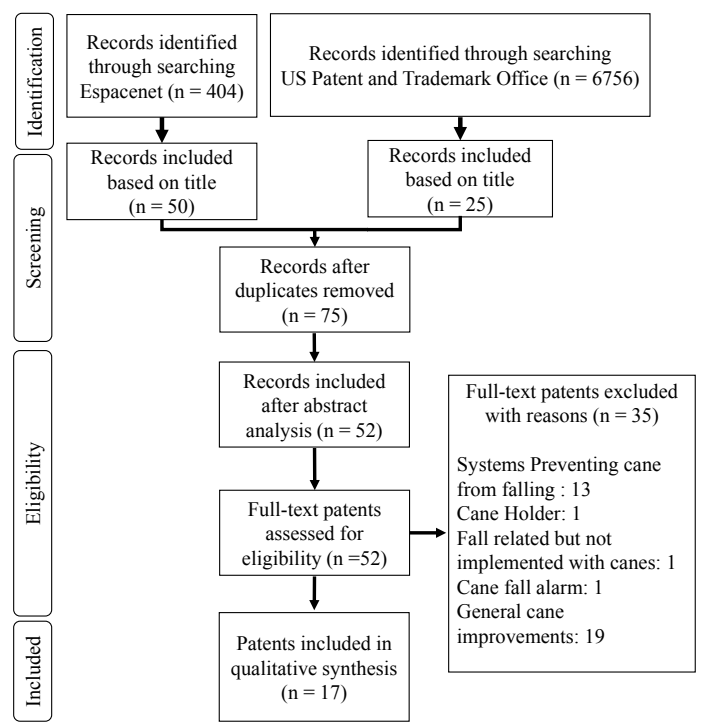

Fig. 2: Flow Diagram based on PRISMA flowchart. 
TABLE I: The three most similar patents with name and scheme to criterion previously established

\begin{tabular}{cccc}
$\begin{array}{c}\text { Name, } \\
\text { Ref }\end{array}$ & Anti-falling walking stick for old & Robotic cane devices & Walking Support Device and \\
person [22] & [23] & Fall Prevention Method [24] \\
\hline Scheme & &
\end{tabular}

\section{CONCLUSION}

The number of prescribed walking aids have been increasing due to gait or balance disorders, lower limbs weakness and their relatively low cost. Thus, the cane is an ideal candidate for universal healthcare and the implementation of fallrelated mechanisms incorporating fall detection, prediction and prevention methods.

After a thorough, careful and comprehensive search, it can be concluded that till this day, it has not been developed a cane system that focuses on the temporal window that precedes a fall. A system that can prevent/minimize a fall by predicting it or through the assessment of the subjects fall risk in real time. The only systems with embedded fall prediction mechanisms are robotic canes and the remaining systems only focus on fall detection. Regarding commercial canes, there is very little supply since only one was available for sale and embeds just fall detection mechanisms. As far as patents go, from the three selected, all present fall prediction mechanisms but solely one does not incorporate an omniwheeled base.

Several steps have already been taken into the development of a cane with fall prediction functionalities. Yet, there is still a need to combine the monitoring potential of a cane with the inclusion of artificial intelligence and the assessment of the risk of falling in real time.

\section{REFERENCES}

[1] World Health Organization, "Falls - WHO," 2018. [Online]. Available: http://www.who.int/news-room/fact-sheets/detail/falls [Accessed: 01Nov-2018].

[2] E. R. Burns, J. A. Stevens, and R. Lee, "The direct costs of fatal and non-fatal falls among older adults United States," Journal of Safety Research, vol. 58, pp. 99-103, 2016.

[3] C. Y. Hsieh, K. C. Liu, C. N. Huang, W. C. Chu, and C. T. Chan, "Novel hierarchical fall detection algorithm using a multiphase fall model," Sensors (Switzerland), vol. 17, no. 2, 2017.

[4] G. C. Chen, C. N. Huang, C. Y. Chiang, C. J. Hsieh, and C. T. Chan, "A reliable fall detection system based on wearable sensor and signal magnitude area for elderly residents," Lecture Notes in Computer Science (including subseries Lecture Notes in Artificial Intelligence and Lecture Notes in Bioinformatics), vol. 6159 LNCS, pp. 267-270, 2010.

[5] A. Lachtar, T. Val, A. Kachouri, A. Lachtar, T. Val, and A. Kachouri, "3DCane : a monitoring system for the elderly using a connected walking stick," international Journal of Computer Science and Information Security, vol. 14, no. 8, pp. 1-8, 2017.

[6] R. Rajagopalan, I. Litvan, and T. P. Jung, "Fall prediction and prevention systems: Recent trends, challenges, and future research directions," Sensors (Switzerland), vol. 17, no. 11, pp. 1-17, 2017

[7] K. L. Perell, A. Nelson, R. L. Goldman, S. L. Luter, N. Prieto-Lewis, and L. Z. Rubenstein, "Fall risk assessment measures: An analytic review," Journals of Gerontology - Series A Biological Sciences and Medical Sciences, vol. 56, no. 12, pp. 761-766, 2001.
[8] T. Xu, Y. Zhou, and J. Zhu, "New Advances and Challenges of Fall Detection Systems: A Survey," Applied Sciences, vol. 8, no. 3, p. 418, 2018.

[9] G. Shi, C. S. Chan, W. J. Li, K. S. Leung, Y. Zou, and Y. Jin, "Mobile human airbag system for fall protection using mems sensors and embedded SVM classifier," IEEE Sensors Journal, vol. 9, no. 5, pp. 495-503, 2009.

[10] H. Bateni and B. E. Maki, "Assistive devices for balance and mobility: Benefits, demands, and adverse consequences," Archives of Physical Medicine and Rehabilitation, vol. 86, no. 1, pp. 134-145, 2005.

[11] C. Luz, T. Bush, X. Shen, and R. Pruchno, "Do canes or walkers make any difference? nonuse and fall injuries," Gerontologist, vol. 57, no. 2, pp. 211-218, 2017.

[12] P. Di, Y. Hasegawa, S. Nakagawa, K. Sekiyama, T. Fukuda, J. Huang, and Q. Huang, "Fall detection and prevention control using walkingaid cane robot," IEEE/ASME Transactions on Mechatronics, vol. 21, no. 2 , pp. $625-637,2016$

[13] P. Di, J. Huang, S. Nakagawa, K. Sekiyama, and T. Fukuda, "Fall detection and prevention in the elderly based on the ZMP stability control," Proceedings of IEEE Workshop on Advanced Robotics and its Social Impacts, ARSO, pp. 82-87, 2013.

[14] P. Di, J. Huang, S. Nakagawa, and K. Sekiyama, "Fall detection for the elderly using a cane robot based on ZMP estimation," 2013 International Symposium on Micro-NanoMechatronics and Human Science, MHS 2013, 2013.

[15] P. Di, J. Huang, K. Sekiyama, and T. Fukuda, "Motion control of intelligent cane robot under normal and abnormal walking condition," Proceedings - IEEE International Workshop on Robot and Human Interactive Communication, pp. 497-502, 2011.

[16] Q. Yan, J. Huang, and Z. Luo, "Human-robot coordination stability for fall detection and prevention using cane robot," 2016 International Symposium on Micro-NanoMechatronics and Human Science, MHS 2016, no. 1, 2017.

[17] M. Lan, A. Nahapetian, A. Vahdatpour, L. Au, W. Kaiser, and M. Sarrafzadeh, "SmartFall: An Automatic Fall Detection System Based on Subsequence Matching for the SmartCane," Sensors Peterborough NH, p. 8, 2009.

[18] P. H. Chen, Y. H. Li, C. W. Chiou, C. Y. Lee, and J. M. Lin, "A smart safety cane for human fall detection," International Journal of Ad Hoc and Ubiquitous Computing, vol. 20, no. 1, pp. 49-65, 2015.

[19] O. Almeida, M. Zhang, and J. C. Liu, "Dynamic fall detection and pace measurement in walking sticks," Proceedings - 2007 Joint Workshop on High Confidence Medical Devices, Software, and Systems and Medical Device Plug-and-Play Interoperability, HCMDSS/MDPnP 2007, pp. 204-206, 2007.

[20] "iStand Smartcane," 2018. [Online]. Available: https://istandtoday.com/ [Accessed: 01-Nov-2018].

[21] "Dring Smartcane," 2018. [Online]. Available: https://dring.io/11canne-connectee-fayet. [Accessed: 01-Nov-2018].

[22] G. Li, Y. Yan, D. Shitong, K. Jie, Y. Zhaoyang, C. Zhanglong, W. Zhen, H. Peng, P. Su, and W. Yaming, "Anti-falling alarm intelligent walking stick for old people," .China Patent 104273812A, Oct.23, 2014.

[23] Y. Ota, M. Ryumae, K. Sato, and S. Sano, "Robotic cane devices," .U.S. Patent 20130041507 A1, Feb.14, 2013.

[24] T. Fukuda and A. Ichikawa, "Walking support device and fall prevention method,” Japan Patent 20140103331, Dec.7, 2015. 